\title{
Co-existence of NCDs and NTDs -trypanosomiasis as a typical example
}

\begin{abstract}
World Health Organization uncover that deaths worldwide from non-communicable diseases (NCDs) exceed those from diseases caused by infections. However, the coexistence of NCDs with pathogenic infection of neglected tropical diseases (NTDs) such as Human African Trypanosomiasis may also contribute to the number of deaths caused by NCDs. NTDs bear similar properties to the NCDs which are their frequent existing and elusive medical signs, and the consequence deformity which last for long. The connection of NCDs and neglected tropical diseases (NTDs) is newly noticed area for scientists to examine. This will open opportunity to examine and control NCDs as most of these diseases are widely found among individuals having NTDs as confirmed by the regular reports obtained from local health care centers of their existence. More research works are required before scientists can say the outcome of co-existence observed in NTDs is NCDs and vice versa. The essence of this paper is to identify and ascertain the existence of close relationship between NTDs and NCDs and to arouse interest of researchers towards this path.
\end{abstract}

Keywords: NCDs, NTDs, coexistence, human African trypanosomiasis, infection
Volume 8 Issue 4 - 2019

\section{Olanrewaju Roland Akinseye}

Nigerian Institute for Trypanosomiasis \& Onchocerciasis Research, Nigeria

Correspondence: Olanrewaju Roland Akinseye, Nigerian Institute for Trypanosomiasis \& Onchocerciasis Research, Nigeria,Email akinseyeroland@gmail.com

Received: June 15, 2019 | Published: July 08, 2019

\section{Introduction}

WHO estimations, uncover that deaths worldwide from noncommunicable diseases (NCDs) exceed those from diseases caused by infections. ${ }^{1}$ Among the major NCDs that cause the highest portion of mortality and deformity in the whole world include chronic heart disease, hypertension, and diabetes mellitus. In total, the conditions recorded to be $50 \%-60 \%$ cause of all mortality globally. As reported by WHO, ${ }^{1}$ most of the NCD mortality occurs in developing nations. ${ }^{2}$ NCDs can be defined as diseases or conditions which do not have pathogen that can move them from diseased person to others but they affect people for a long time. ${ }^{2}$

Among all other diseases caused by pathogenic infection, the neglected tropical diseases (NTDs) such as Human African Trypanosomiasis bear similar property to the NCDs. The properties are their frequent existing and elusive medical signs, and the consequence deformity lasting for long. A poor individual that has been infected with a NTD could carry the disease for long time or even the whole entire life. ${ }^{3,4}$ Apart from the pathogenic agents that cause disease, the NTDs fit into the NCDs description characteristics.

The connection of NCDs and neglected tropical diseases (NTDs) is newly noticed area for scientists to examine. The initial stage detection has double benefits over final detection and control of NTDs. This might open opportunity to examine and control NCDs as most of these diseases are widely found among individuals having NTDs as confirmed by the regular reports obtained from local health care centres of their existence. ${ }^{5}$ More research works are required before scientists can say the outcome of co-existence observed in NTDs is NCDs. The reason is that the diseased condition of NCDs seen in NTDs is very far from detail information required. At the final step of its prediction, NCDs resulted from NTDs, though might be caused by other means, which could be difficulties come from delay in seeking treatment by infected person or conditions already in the final step of manifestation. ${ }^{5}$
Precisely, in relation to the populations not given proper health attention, neglected tropical diseases (NTDs) together with other poorrelated disease agent might be the core cause of chronic heart disease and other cardiovascular disease. According to data estimates from WHO that analyzed the Global Burden of Disease, cardiovascular disease might be responsible for $8.8 \%$ deformity in less developed nations. ${ }^{6}$ Prolong heart disease suggested to result from constant presence of trypanosome in the hepatic tissue, which result in diverse destruction of tissue, dilation of vein system and abnormal increase of fibrous tissue. ${ }^{7}$ Inflammation of myocardium and pericardium has been attributed to cause by Human African trypanosomiasis (HAT) during the severe step of the conditions when the infective stages (trypamastigote) of the microorganism extend to cause inflammation of the inner lining of artery through blood and lymph. ${ }^{8}$

\begin{tabular}{ll}
\hline $\begin{array}{l}\text { NCDs Chronic } \\
\text { Condition }\end{array}$ & $\begin{array}{l}\text { NTDs as } \\
\text { Etiology }\end{array}$ \\
\hline Chronic heart failure & HAT \\
Anaemia & HAT \\
Diabetes & Not determined \\
\hline
\end{tabular}

\section{HAT, Human African Trypanosomiasis}

Anaemia is a health condition that can be observed in lowincome, middle-income and high-income countries at every age. It occurs when the hemoglobin $(\mathrm{Hb})$ levels is greater than $12.0 \mathrm{~g} /$ $\mathrm{dL}$ in women and $13.0 \mathrm{~g} / \mathrm{dL}$ in men. Anaemia occurs in humans, livestock and experimental mouse models. In humans, anaemia is the common characteristic during the haemolymphatic stage; it is the main diagnostic tool, followed by parasite detection in the blood. The anaemia of trypanosomiasis usually sets in during the first wave of parasitaemia although some workers did not observe anaemia in early phase of human Trypanosomiasis. ${ }^{9}$

Subsequent development of the anaemia is determined by the frequency and intensity of parasitaemia. ${ }^{9}$ Several erythrocyte 
morphological abnormalities observed in trypanosomiasis infection of red blood cells include anisocytosis, poikilocytosis, polychromasia, punctuate basophilia, macrocytosis, microcytosis, spherocytosis and schistocytosis have been describes, in various combinations, in $T$. brucei and $T$. congolence infections ${ }^{9}$ and in human $T$. rhodesiense infection. ${ }^{9}$ Hence, anaemic conditions meet the criteria of noncommunicable diseases that co-exist and result from neglected tropical diseases.

Infection caused by trypanosomiasis has not been reported to lead to any type of diabetes sickness, but previous studies, the frequent sign and symptoms consistent with cardiac malfunction which include arterial hypotension, palpitations, thoracic pain, exertional dyspnoea, and hepatomegaly are observed in patients infected with $\mathrm{T}$. b. gambiense HAT than healthy controls. ${ }^{10-12}$

However, there has not been study about the co-morbidity of Type 2 diabetes with Human African Trypanosomiasis caused by T. brucei gambiense. African trypanosomiasis is a general menace in subSaharan Africa. ${ }^{13,14}$ Type 2 diabetes mellitus (T2DM) is an increasingly common disorder characterized by chronic hyperglycemia and marked dyslipidemia. ${ }^{15,16}$ Trypanosomiasis has immuno-suppressive ability which could enable them hide or accommodate any other disease. During trypanosomiasis, nonspecific triggers of lymphocytes ( $\mathrm{T}$ and $\mathrm{B}$ ) united with immuno-suppression, provide means for the trypanosome to evade, survive, and transmit. ${ }^{17,18}$ Type 2 diabetes is generally observed to be inflammatory disease that results from prolonged immune instability, metabolic abnormality or excessive body weight cause by too much nutrients. ${ }^{19-21}$

The hypothesis that the excess glucose observed in the case of diabetes mellitus could be the source of food where HAT derived its energy needed for binary fission and to drive antigenic variation manipulation mechanism should be investigated. The ability of one disease to feed on the products of another could establish a parasitic, symbiotic or commensals association and could hide the disease in the body for a long period of time.

\section{Conclusion}

Conclusively, this review is design to expose the connection of NCDs, (such as cardiovascular heart diseases, anaemia, and diabetes mellitus) with the underlying NTDs. NTDs could either be the cause of NCDs or result from other source while both conditions co-exist in the same host. The essence of this paper is to identify and ascertain the existence of close relationship between NTDs and NCDs and to arouse interest of researchers towards this path.

\section{Acknowledgements}

None

\section{Conflicts of interest}

The author declares that there are no conflicts of interest.

\section{References}

1. World Health Organization. Major NCDs and their risk factors. Geneva: World Health Organization (WHO); 2018.

2. Daar AS, Singer PA, Persad DL, et al. Grand challenges in chronic noncommunicable diseases. Nature. 2007;450(7169):494-496.
3. Hotez PJ, Molyneux DH, Fenwick A, et al. Control of neglected tropical diseases. N Engl J Med. 2007;357(10):1018-1027.

4. Hotez PJ, Molyneux DH, Fenwick A, et al. Incorporating a rapid-impact package for neglected tropical diseases with programs for HIV/AIDS, tuberculosis and malaria. PLoS Med. 2006;3(5):e102.

5. Mishra SR, Dhimal M, Bhandari PM, et al. Sanitation for all: the global opportunity to increase transgenerational health gains and better understand the link between NCDs and NTDs, a scoping review. Trop Dis Travel Med Vaccines. 2017;3:8.

6. World Health Organization. Projections of mortality and burden of disease, 2004-2030; standard DALYs (3\% discounting, age weights) - baseline scenario. Geneva: World Health Organization (WHO); 2012.

7. Benvenuti LA, Freitas HFG, Mansur AJ, et al. Chronic American trypanosomiasis: parasite persistence in endomyocardial biopsies is associated with high-grade myocarditis. Ann Trop Med Parasitol. 2008;102(6):481-487.

8. Barratt JLN, Harkness J, Marriott D, et al. Importance of nonenteric protozoan infections in immunocompromised people. Clin Microbiol Rev. 2010;23(4):795-836.

9. Anosa VO. Haematological and biochemical changes in human and animal trypanosomiasis. Revue Elev Med Vet Pays trop. 1988;41 (1):65-78

10. Blum JA, Burri C, Hatz C, et al. Sleeping hearts: the role of the heart in sleeping sickness (human African trypanosomiasis). Trop Med Int Health 2007;12(12):1422-32.

11. Blum JA, Schmid C, Burri C, et al. Cardiac Alterations in Human African Trypanosomiasis ( $\mathrm{Tb}$ gambiense) with Respect to the Disease Stage and Antiparasitic Treatment. PLoS Negl Trop Di. 2009;3(2):e383.

12. Kristensson K, Nygård M, Bertini G, et al. African trypanosome infections of the nervous system: parasite entry and effects on sleep and synaptic functions. Prog Neurobiol. 2010;91(2):152-71.

13. Abenga JN, LA Lawal. Implicating role of animal reservoir host in the resurgence of Gambian trypanosomiasis (sleeping sickness). Air I Biotech. 2005;4(2):134-137.

14. Chreihen JPL, BL Smoak. African Trypanosomiasis:Changing epidemiology and consequences. Curr Intec Dis Reports. 2005;7(1):54 60.

15. Vinson JA, Zhang J. Black and green teas equally inhibit diabetic cataracts in a streptozotocin-induced rat model of diabetes. J Agric Food Chem. 2005;53(9):3710-3713

16. Bhattacharya S, Dey D, Roy SS. Molecular mechanism of insulin resistance. J Biosci. 2007;32(2):405-413.

17. Vincendeau P, Bouteille B. Immunology and immunopathology of African trypanosomiasis. An Acad Bras Cienc. 2006;78(4):645-665.

18. Edwin Garzón, Philippe Holzmuller, Rachel Bras-Gonçalves, et al. Trypanosoma brucei gambiense secretome impairs LPS-induced maturation, cytokine production, and the allostimulatory capacity of dendritic cells. Infect Immun. 2013;81(9):3300-3308.

19. Guzman-Flores JM, Lopez-Briones S. Cells of the innate and adaptive immunity in type 2 diabetes mellitus and obesity. Gac Med Mex. 2012;148(4):381-389.

20. Shu CJ, Benoist C, Mathis D. The immune system's involvement in obesity-driven type 2 diabetes. Seminars in Immunology. 2012;24(6):436442 .

21. Rodak BF. Hematology: clinical principles and applications. Philadelphia: Saunders Publications: 2007. p. 220. 